\title{
Central Themes of the Pokémon Franchise and why they Appeal to Humans
}

\author{
Samuli Laato \\ University of Turku \\ sadala@utu.fi
}

\author{
Sampsa Rauti \\ University of Turku \\ sjprau@utu.fi
}

\begin{abstract}
Pokémon has become the most profitable cross-media franchise since its creation 25 years ago. In this conceptual work, we go through the current eight generations of Pokémon games, movies and anime episodes to understand why the franchise appeals to humans. We focus on the following four core themes: (1) relationship with the Pokémon creatures; (2) exploration and adventure; (3) social connectedness and acceptance; and (4) green technologies and living in symbiosis with the environment. We discuss how these themes resonate with human primal desires and what aspects of Pokémon transcend into the real world. Our findings indicate that the Pokémon world is able to offer humans aspects of the hunter-gatherer era that are missing from the current urban way of living. The franchise appeals to human primal desires by being about exploring the world, understanding it and finding harmony with other living creatures. Our findings support previous work on the appeal of specific Pokémon games, and contributes to our understanding of what has made the franchise popular.
\end{abstract}

\section{Introduction}

According to TitleMax, during its first 23 years of operation, the Pokémon franchise grossed over 90 billion USD [1] making it the most profitable cross-media franchise of all time. Being first introduced to the public via GameBoy video games Red and Blue, Pokémon exploded into a global phenomenon between 1998-2001, after which its popularity saw a slight decline [2]. Yet, the franchise maintained a large fandom with new pokémon anime (animated series) episodes, games, movies, trading card sets, manga and merchandise released at a steady pace. In 2016 the launch of Pokémon GO rekindled the popularity of Pokémon and gave birth to a new franchise renaissance with new cinematic movie releases (e.g. Detective Pikachu and Mewtwo Strikes Back: Evolution), spin-off games (Pokémon Let's GO -series) and main series games (Pokémon Sun \& Moon and Sword \& Shield) selling record-breaking numbers [3]. As of June 2020, there are over 100 different Pokémon games including spin-offs, as well as countless other products and merchandise.

Recently studies have been conducted on why people play individual Pokémon games, (e.g. Pokémon GO [4, $5,6])$. While these studies have provided insights into player engagement with specific games, they have failed to account for story elements that are characteristic to the Pokémon franchise beyond broad constructs such as "nostalgia" [5]. In order to study the role of the story and imagination aspects of Pokémon in the playing experience, a thematic analysis of Pokémon franchise products is needed. The aim of the current study is to identify core themes of the Pokémon franchise as a whole, to see why people stay engaged with it beyond individual games. We argue this kind of an analysis can offer insights into what has made Pokémon the most profitable cross-media franchise of all time. Furthermore, findings can offer viewpoints into the fields of gamification and game design, helping designers understand the role of stories and fictional worlds in the playing experience.

In the current study we refer to the fictional universe that Pokémon products build as the Pokémon world. Through analysis of Pokémon franchise products including games, animated series and movies, as well as research on these three, we extract the following as central repeating themes: (1) relationship with the Pokémon creatures [7]; (2) exploration and adventure; (3) social connectedness and acceptance; and (4) green technologies and living in symbiosis with the environment [8]. In order to understand why these themes apply to humans, we take an analysis lens of what the human brain has evolved to desire, and then compare how the themes can address these desires. Therefore, as a framework for theoretical reasoning and analysis, we utilize the human primal desires perspective as guided by Panksepp [9]. We continue this analysis by 
looking at features of the Pokémon world that extend into our world such as link cable trading that encourage players to engage in face-to-face banter in order to agree on action in the game world. Finally, we compare our analysis with other work and detail the limitations of the current study.

\section{The design and history of Pokémon}

In 1991, a Japanese game designer Tajiri Satoshi came up with the concept of Pokémon [8]. Tajiri envisioned a utopian world inhabited by creatures called Pokémon, reportedly drawing influence from his bug collection hobby, the Yokai in Japanese folklore and Godzilla movies [10]. Based on his ideas, two video games (Pokémon Red and Blue) were developed for the then current Nintendo flagship mobile gaming console GameBoy, and were released in Japan in late 1996, and later to the rest of the world. These games laid a foundation to the cross-media franchise that would turn into a global phenomena in the next three years. Currently eight generations of Pokémon games have been published, with the games introducing a new generation being listed in chronological order in Table 1 .

Table 1. A list of main series Pokémon games.

\begin{tabular}{lcc}
\hline Game & Released & Platform \\
\hline Red/Blue & 1996 & GameBoy \\
Silver/Gold & 1999 & Gameboy Color \\
Ruby/Sapphire & 2002 & Gameboy Advance \\
Diamond/Pearl & 2006 & Nintendo DS \\
Black/White & 2010 & Nintendo DS \\
X/Y & 2013 & Nintendo 3DS \\
Sun/Moon & 2016 & Nintendo 3DS \\
Sword/Shield & 2019 & Nintendo Switch \\
\hline
\end{tabular}

The majority of Pokémon products have always been aimed at a child audience, which is evident from, for example, the visual appearance of characters and their actual reported age [10]. Ads for both Pokémon games and other products also typically feature children enjoying the products. While the Pokémon anime series contains story-elements that are typical to kids cartoons such as a repeating events (e.g. Team Rocket has an evil plan, they almost succeed but the heroes prevail in the end), the Pokémon movies contain deeper and more varied themes including (1) do clones have the same value as their original counterparts (Mewtwo Strikes Back: Evolution); and (2) can nature be contained and captured (Pokémon The Movie 2000). The main storyline of Pokémon games is typically straightforward, with the notable exception of Pokémon Black/White that deals with the moral dilemma of capturing pokémon. The same theme is also reflected upon in the fourth season of the Pokémon anime, "Pokémon the series: Black and White". Individual Pokémon anime episodes have changing themes which include motherly care (e.g. "First Catch in Alola, Ketchum-Style!" episode of the Sun and Moon Pokémon series), overcoming bullying (e.g. episode 9 of the Indigo League series) and whether pokémon can be evil or not (e.g. episode 17 of the Indigo League series).

Pokémon GO brought a change in the Pokémon franchise by modelling the main character as a distinctive adult. This was arguably done to appeal to an older audience. Pokémon GO was downloaded over 500 million times during its launch year and quickly became one of the highest grossing video games ever [5]. Before Pokémon GO, the extant Pokémon literature viewed the franchise primarily from the perspective of children's media culture [11]. However, as a significant amount of active Pokémon GO players are adults (e.g. [4]), and popular Pokémon game players and speedrunners are adults (e.g. Werster) [12], it seems that the Pokémon world keeps engaging adults as well. Previous work on why people engage with the pokémon products have found nostalgia to indeed play a role [5, 13], but also many other reasons have been given, such as Pokémon being perceived by consumers as a cool brand [14]. These findings invite a more careful analysis into the fictional and thematic elements of the Pokémon franchise.

\section{The Core Themes of Pokémon}

The Pokémon world can be seen to have four major themes that are present in both the games and the anime. The first is the relationship between a trainer and their pokémon [15]. The second is interaction with nature, including exploration, adventure, collection and observation $[8,15]$. The third is society in the form of inter-human relationships, trading, barter and social belonging $[8,16]$. The fourth one is future technology, and how technology should enable a harmonious living among other lifeforms (pokémon) and nature. Together, these themes shape and define the Pokémon world. In the following, we discuss each of these four themes in detail, how they are present in Pokémon games and how they are expanded upon. Our aim is to understand why they appeal to humans and what we can learn from ourselves through Pokémon.

\subsection{The trainer and their pokémon}

"I wanna be the very best, like no one ever was. To catch them, is my real test - to train them is my cause.” [17] 
In Pokémon franchise games and movies, consumers typically assume the role of a trainer, that is, someone who captures, trains and battles with pokémon [15]. In the main series Pokémon games the player controls a young new trainer who receives their first pokémon and embarks on an adventure. In the Pokémon movies, anime series and manga, the story typically follows a group of trainers [10]. The pokémon trainer is someone whose goal is to explore the world, discover (all) new pokémon, capture them, train them and win pokémon battles. The main series pokémon games (Table 1) offer trainers all sorts of additional goals and things to do ranging from pokémon breeding to participation in berry blending and beauty contests. However, the player always controls a pokémon trainer who travels the world accompanied by their pokémon.

The relationship between a pokémon trainer trainer and (their) pokémon is a central theme that is present to a degree in almost all Pokémon franchise games. The theme is explored from various viewpoints throughout the franchise, and many other more minor themes are connected to it. The biophilia hypothesis posits that humans have a natural desire to get to know and spend time with other lifeforms [18], and scholars have argued that the Pokémon franchise is able to invoke this desire [7]. Indeed, when playing Pokémon games it becomes clear that the narrative supports a symbiotic, caring relationship with pokémon. For example, upon completing the first Pokémon game Red/Blue, the player is told to have conquered all challenges because of a loving relationship with pokémon, and that the main rival lost the final battle as he failed in doing so. In Pokémon Black/White the anti-hero character "N" has an agenda to free all pokémon as he believes capturing and enslaving them to be unethical. In the movie Pokémon: The First Movie: Mewtwo Strikes Back the main character Ash Ketchum convinces a powerful Pokémon Mewtwo that he is partners with his first pokémon Pikachu rather than considering Pikachu a slave. In the second Pokémon Movie, Pokémon The Movie 2000: The Power of One, the main antagonist Lawrence the Second attempts to capture and enslave four legendary bird Pokémon which represent the forces of nature. As the antagonist proceeds to capture the legendary birds by force, the world is thrown into chaos. The conflict is resolved when the legendary birds i.e. the forces of nature are released and symbiosis between humankind and pokémon is re-established. This can be seen as a metaphor of what will happen if humans abuse nature instead of partnering with it. The theme of biophilia is present in Pokémon The Movie 2000: The Power of One in the comparison of how the main protagonist Ash Ketchum keeps his partner Pokémon
Pikachu by his side, free of constraints, while the main antagonist Lawrence the Second keeps captured Pokémon in cages against their will.

Perhaps contradictory to these themes of partnership with nature, biophilia and natural harmony stands capturing pokémon and pokémon battles. Pokémon battles are central in the pokémon series and are the primary way to resolve conflicts. Figure 1 depicts how the trainer is often visualized in Pokémon games to be on the immediate background of the pokémon battle. The same applies in Pokémon movies and the anime. As such, pokémon battles are depicted as fights between humans that use pokémon as a proxy. This draws parallels to sports and board games such as chess where humans engage in conflict through a playful set of agreed rules. Pokémon franchise products seem to stress on multiple occasions that pokémon do not mind battling, and that it is in fact natural and they even enjoy it (e.g. Sun \& Moon anime series, ep. 15). In the Pokémon Indigo League anime, the main protagonist Ash Ketchum is shown on multiple occasions to even take hits for his main partner pokémon Pikachu, and only battling with Pikachu on the pokémon's terms. The narrative of the Pokémon world highlights that pokémon are not to be used as tools for battle, or to be captured and enslaved, but instead, seen as partners. This mirrors, for example, horse owners who have been reported to strive for a partnership with their steeds [19].

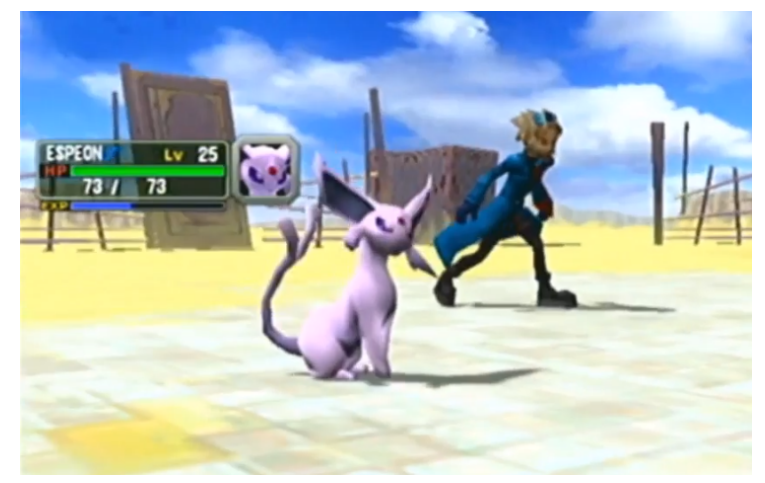

Figure 1. Typical visual feedback of a Pokémon battle. Screenshot taken from the game Pokémon Colosseum by the authors.

Pokémon battles are also the primary way the trainer "trains" their pokémon. However, more recent games (e.g. Pokémon Sword/Shield) have also introduced other mechanics to train and level up pokémon such as simply being in the trainer's party or camping and cooking with the trainer. The primary goal of training a pokémon is to make it more powerful, and by extension, make the trainer more powerful. In addition, pokémon also grow to like their trainer. The bond between the 
trainer and their pokémon is supported and built by visual and story elements. For example, some pokémon such as Chansey and Togepi only evolve to stronger forms when they have reached a loving bond with their trainer. Certain pokémon battle moves such as "return" deal increased damage the stronger the bond between the pokémon and the trainer is. In many of the later games (e.g. Let's GO Eevee/Pikachu and Pokémon GO) pokémon gain even further bonuses from bonding with their trainer such as chances to automatically heal status conditions in battle or helping in capturing other wild pokémon. In the Pokémon anime series, training a pokémon encompasses much more than battling. The pokémon participate in basic activities with their trainer such as eating, sleeping and even decision making.

To summarize, the Pokémon franchise strongly supports the idea of partnering up with pokémon instead of using them as tools or enslaving them. Formation of the partnership is supported by game mechanics as well as narrative elements. The idea that pokémon are not tools to be exploited, but fascinating creatures, is present in almost all Pokémon games and movies to some extent, and is the main focus in, for example, the very first Pokémon movie and the first Pokémon games. As such, the Pokémon franchise seems to embrace the biophilia hypothesis [7, 18] which states that humans have a natural tendency to spend time with other creatures and get to know them. The success of the Pokémon franchise provides support to the idea that humans prefer to partner with other living creatures rather than use them as tools.

\subsection{Adventure, exploration and nature}

"I will travel across the land, searching far and wide, each pokémon to understand, the power that's inside” [17]

The Pokémon world motivates exploration via beautiful scenery and story. Typical pokémon games contain various landscapes such as forests, deserts, oceans, rocky mountains, tunnels and caves, beaches, small towns, large cities, busy roads, lonely roads and all kinds of other places that can be explored. This is typical to adventure and RPG games such as the Elder Scrolls V: Skyrim and the upcoming Cyberpunk 2077 [20]. While individual Pokémon games are limited by the number of areas and their size, the games suggest that there is an endless amount of landscapes to explore in the Pokémon world. This way the narrative provides players the incentive to purchase additional Pokémon franchise products. Pokémon GO was a game changer among previous Pokémon games in that it transformed the real world into a playground which created a practically endless amount of grounds to explore [21]. Supporting our analysis, studies have found that a significant portion of Pokémon GO players are motivated specifically by the exploration aspect [6].

Recently the Pokémon development team has drawn inspiration from real world countries to design areas and culture in games. Pokémon $\mathrm{X} / \mathrm{Y}$ were based on the culture of France, Sun/Moon were based on the culture of Hawaii and Sword/Shield were based on the culture of the United Kingdom. Also inside individual games, different cultures are presented. Sword/Shield, for example, depicts small town life, busy city life, gang life and rich posh life. The NPCs (non-playable characters) in the game typically represent some real life occupation and have an identity which determines how they look. Possible trainer identities involve hikers, schoolboys, engineers, gang members and fishermen among others. Only special characters such as gym leaders and those directly connected to the story typically have their own unique appearance. Taken together, it is not only landscapes that the games allow players to explore, but cultures as well. The wide range of territory and cultures gives an impression of a vast world filled with mystery.

The theme of exploration, adventure and venturing into the unknown is balanced and countered by capturing and collecting pokémon, which represents on a larger thematic level the human desire to contain and control nature [8, 10]. This tension between conversing with the unknown (exploration) and making peace with it (understanding) manifests throughout the Pokémon world in gameplay and in the story. Players are encouraged to go out and explore, but nature is dangerous and their pokémon can get hurt. In Pokémon games the game mechanics only allow the trainer to explore the lands as long as they have healthy pokémon with them, highlighting the importance of the relationship between the trainer and their pokémon we discussed earlier. The trainer can always return to familiar pokémon centers (displayed in Figure 2) where pokémon can be healed for free. The presence of these safe havens further encourage exploration, as there is a safe place to retreat to.

In order for players to be engaged with exploration of the Pokémon world, they need to perceive the world to be meaningful. Perhaps the most omnipresent motivation in the Pokémon franchise to motivate exploration is "gotta catch 'em all" i.e. capture all unique pokémon species [11], which is emphasised by strategic placement of endemic pokémon across the world. As an example, electric pokémon can be found in power plants, poison type pokémon can be found in places with contamination, water pokémon can be 


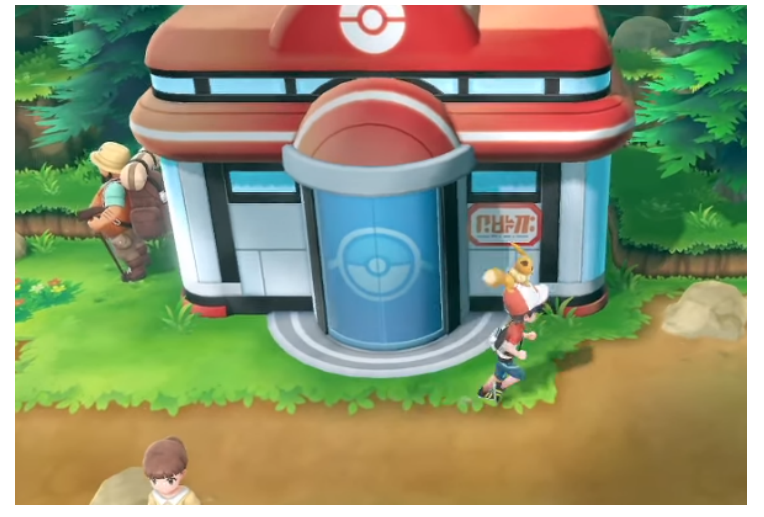

Figure 2. Displaying a pokémon center. Screenshot taken by the authors from the game Let's GO Eevee.

found in the sea and ground pokémon typically live underground in tunnels. Pokémon are also depicted to live in their own ecological niches (e.g. Muks and Garbodors devour waste, Corsola clean up oceans). These factors make the Pokémon world seem like a utopian version of our world where pokémon have evolved to counter the deficits of the modern way of living and polluting. The inclusion of real biological concepts in the design of the Pokémon world makes it seem more realistic, supporting imagination. Indeed, the idea of pokémon being real made itself a part of children's playing quite soon after the release of the first Pokémon franchise products $[8,22]$. This shares similarities with many other popular franchises such as Harry Potter (what if wizards were real), Star Wars (what if the force was real) and Dragon Ball Z (what if I had super-human power and speed).

Finally, Pokémon games and anime also contain several mysteries, which arouse curiosity, a feeling related to exploration. Thus, the player is not only prompted to explore nature, but also to solve mysteries and learn to understand the Pokémon world. This central theme is supported by gameplay such as field research tasks in Pokémon GO where the player is asked to perform playful tasks masked as research, and also mystery solving such as the Looker Bureau missions in Pokémon $\mathrm{X} / \mathrm{Y}$ where the player is tasked to resolve a series of mysterious detective cases. Solving these mysteries can be connected to learning, and Elza argues that learning and personal growth is an important cross-cutting theme in the Pokémon franchise that connects to exploration [22]. The Pokémon world can thus also be considered an active learning environment where trainers learn through personal exploration and investigation.

\subsection{Human relationships and friendship}

"Pokémon! It's you and me. I know it's my destiny, Pokémon! Oh, you're my best friend, In a world we must defend!'” [17]

The creator of the Pokémon Franchise, Satoshi Tajiri, is said to have been inspired by the Game Boy link cable technology that allowed two players to connect their devices together [10]. Tajiri's ideas manifested in the first Pokémon game being split into two, Pokémon Red and Blue, which both had unique pokémon that could not be obtained in the other version without link cable trading. Furthermore, some pokémon, namely Haunter, Graveler, Kadabra and Machoke, required a link trade in order to evolve. The link cable trades brought players physically together and encouraged them to engage into trading and bartering [10]. All main series Pokémon games since then have contained two versions at launch (see Table 1) which have unique pokémon, obtaining which requires trading between the two versions. Until Pokémon X/Y, players needed to get to physical proximity of one another to perform the trade. However, $\mathrm{X} / \mathrm{Y}$ introduced online trading after which connecting with other trainers has been possible from a distance.

Meeting new people and getting to know them is a major part of the Pokémon world's story. In Pokémon games, meeting new characters often begins with a pokémon battle and can also contain slight verbal conflict as depicted in Figure 3. However, the conflict almost always discharges after the battle. This continuous tension between conflict and resolution characterizes the entire Pokémon franchise. In Pokémon Silver/Gold, after trainer battles are over, many NPCs give the player their phone number for future correspondence. As such, pokémon battles act as a metaphor for social anxiety which needs to be overcome in order to form relationships. They can also be seen to teach that fighting and conflict are a natural part of life, that once settled, gives rise to friendships and harmony. The rules of pokémon battles and their role as a conflict resolver are respected throughout the Pokémon world. For example in Red/Blue and Silver/Gold the criminal organization Team Rocket disbands all their activities once the player defeats their leaders in a pokémon battle.

In addition to pokémon battles, characters in the Pokémon world interact in various other ways. These include conversations, berry blending, pokémon trading, shopping and so forth. While in the anime series and movies the main characters travel in groups of trainers, in the main series Pokémon games (see Table 1) the player travels alone only accompanied by pokémon. 


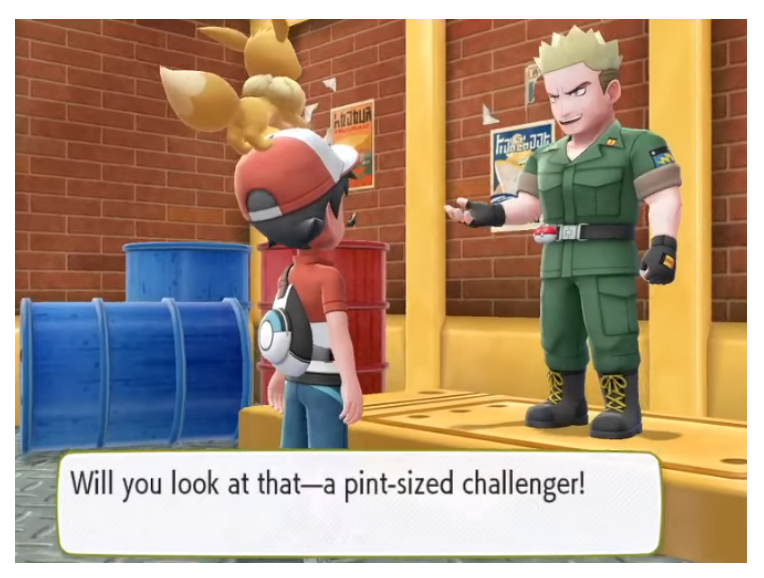

Figure 3. The player challenging the Vermillion city gym leader Lt. Surge. Screenshot taken by the authors from the game Let's GO Eevee.

The level of interaction with others in-game characters or NPCs remains superficial, but especially the later games from $\mathrm{X} / \mathrm{Y}$ onward have started to display the main character to have a group of friends whom the player frequently interacts with. In both Pokémon games and the anime, helping others is a key motivator. Altruistic behaviour is displayed from behalf of the main characters, NPCs, and even the supposed villains. This almost complete lack of social conflict can make players feel more at peace and accepted.

\subsection{Future technology and symbiosis with nature}

Pokémon games, movies and anime are filled with futuristic technology, of which perhaps the most omnipresent are the poké balls that are used to capture and store pokémon. In addition, futuristic looking machines of various sorts are displayed and featured throughout the Pokémon world. These include a complex storage system that transforms pokémon into digital form and keeps them stored and a teleportation fusion system that enables humans to inhabit the bodies of pokémon. These technologies on their own may raise concerns about whether pokémon are being enslaved and abused by technology. On the other hand, futuristic technology is also used to heal wounded pokémon and support their growth. Pokémon were originally introduced as pocket monsters [23], creatures who after initial struggle, are happy to get caught by a trainer. Presenting pokémon as creatures who have different desires and goals from humans can be seen as a way to justify the capturing of pokémon.

Most Pokémon games and the anime feature urban planning that shares the theme of energy conservation.
Regularly Pokémon towns are displayed to have wind turbines and aesthetics combining modern architecture with nature. Examples include a city built on trees (Fortree city, Pokémon Ruby/Sapphire) and a city built around waterfalls (Phenac city, Pokémon Colosseum). This also aligns with the theme of finding balance and equilibrium with the surrounding nature. In the episode 19 of the Indigo League Pokémon series the plot revolves around a character called Nastina who attempts to destroy the natural habitat of a Pokémon species called Tentacool in order to construct a hotel in that place. The episode deals with the consequences of humans destroying nature for their own personal gain. In the episode the Tentacool fight back, and chaos ensues. Once the main characters make peace with the pokémon (representing nature), harmony and peace is achieved yet again. Humans destroying nature is also criticized by pokémon design. Sword/Shield introduced a coral reef pokémon "Corsola" that had been killed by humans, being forced to adapt to living as a ghost type pokémon, changing name to "Cursola".

The Pokémon world features several scientists. The very first games begin by professor Oak giving the player a high tech pokémon encyclopedia, the Pokedex, which the player then sets out to fill. On this journey, the player meets several of Oak's research assistants, a hermit scientist named Bill and laboratory employees on Cinnabar island who are able to revive extinct pokémon from a fossil sample. The presence of science is noticeable in almost all of the follow-up games with them always beginning with a professor giving the player their first pokémon. Figure 4 displays Professor Elm giving the player their pokémon in the game Soul Silver. The presence of science and scientists connects to the theme of learning through exploration and discovery [22]. Some games such as Ruby and Sapphire base their entire plot on the conflict of two groups of mad scientists who seek to take over the world with the help of technology. Pokémon Silver and Gold on the other hand feature a plot where Team Rocket takes control of the Radio Tower. This theme of technology being used for evil is repeated throughout Pokémon games. The solution is always to find peace and an equilibrium with the people, nature, pokémon and technology.

Transportation in the Pokémon world is a mix between green technologies, futuristic design and natural solutions. In the games the main character first moves by foot before acquiring a bike at some point. Further down the road, the player can learn to fly with their pokémon to reach all areas quickly. In Pokémon Omega Ruby / Alpha Sapphire the player is even allowed to control their pokémon as they fly 


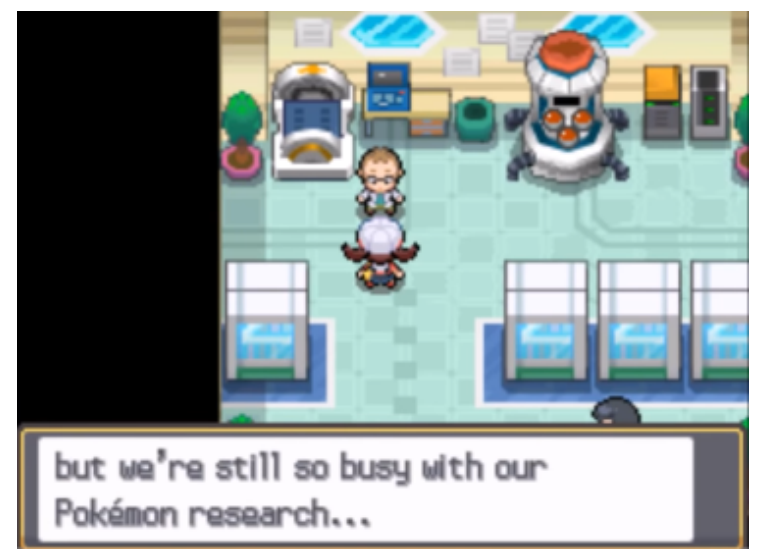

Figure 4. The player converses with the Pokémon professor Elm in his lab. Screenshot taken by the authors from the game Pokémon Soul Silver

around the world. While these are the main methods the players move with, other forms of transportation are featured in games. In Silver/Gold the player takes a boat ride on a large cruise ship and later uses a magnet train. In Ruby/Sapphire the player ascends a mountain using a cable car. The games also feature a space center that displays a rocket resembling the Russian Soyuz-TM model. Furthermore, the Pokémon games feature more sophisticated transportation methods such as a teleportation system (in Silph. co. building and Saffron city gym in Red/Blue). What is common in all these transportation methods is that they are never displayed to be polluting, and are rather depicted as green alternatives. Furthermore, moving with a vehicle is only a secondary option that is done occasionally, and in both the games and the animated series, the characters most often move by walking. The emphasis on walking and adventuring is arguably one of the reasons why Pokémon GO works so well as a physical activity increasing exergame [24].

\section{How Pokémon relates to and stimulates primal instincts and desires}

In order to understand why the Pokémon franchise keeps engaging players, we look at the identified themes through the perspective of affective neuroscience [9]. Affective neuroscience conceptualizes human affects to be the result of three layers of processes: primary, secondary and tertiary [25]. The first layer is deeply subcortical and is shared with most other mammalian species [25]. The second layer operates mostly in the upper limbic system, and this is where memories are stored and where the subconscious resides [9]. Humans have experienced and learned different things, however, the secondary processes are guided by primary processes and society, meaning similarities between humans will emerge. The tertiary layer is for active cognitive processing, and cognitive regulation of learning. The three layers are nested, so that secondary processes reside on top of primary processes and tertiary processes reside on top of both [25]. Therefore, when we discuss primal instincts and desires, we refer predominantly to deeply subcortical emotions and affects. There are seven unique types of primal processes, which following the neurofunctional nomenclature are: (1) seeking; (2) play; (3) care; (4) lust; (5) rage; (6) fear; and (7) panic/grief [25]. All these processes can be connected to affective feelings, seeking to enthusiasm, play to joy, care to tenderness and love and so forth. The first four in the list are positively rewarding feelings, meaning mammals including humans want to experience things that cause these feelings [9].

Seeking, sometimes also called the desire system, is a general purpose motivational system that drives people to seek food and other rewards $[9,25]$. It is responsible for our desire to explore and wonder. Thus, the system connects well to the theme of exploration (theme 2) that is ubiquitously present in the Pokémon world. Players know that this system is in operation when they feel enthusiasm. the Pokémon world being a grand world filled with wonders keeps the seeking system satisfied for long periods of time. If the seeking system is prevented from functioning, the rage system will be aroused. Generally however, players will simply quit playing once the game no longer manages to keep their seeking system satisfied. The seeking system also enables other primal processes such as play, care and lust to operate effectively [9]. The Pokémon world can be regarded as a fictional, playful environment, appealing to the "play" process. Players are able to act out stories, adventures and experiences which brings them joy. The Pokémon world can also stimulate the care system, as pokémon are trained and taken care of by the player. This connects to the first theme, the relationship between the trainer and their pokémon. While the pokémon games are unable to reproduce some aspects of the care system such as touch, they can stimulate the human imagination in ways that arouses the care system. [9, 25]

The identified themes of the Pokémon world can also appeal to human experience. Studies have already shown nostalgia (which is based on memory) to be a central reason players started playing Pokémon GO [4, 5]. We have also evidence that the Pokémon world can invoke several other core desires, which include (1) biophilia [7]; and, desires to (2) live in symbiosis 
and harmony with the environment [8]; (3) explore and control territory; (4) be socially connected and accepted; and (5) be free of constraints. In addition, Pokémon can give social gratification, which can also be regarded as a primal need. Looking at the Pokémon world from an evolutionary psychology perspective, it seems to imitate several aspects of living which are missing from modern urban societies such as: adventure (theme 2), time spent with living creatures (theme 1) and also to an extent, social acceptance (theme 3 ).

\section{Connection between the Pokémon world and the real world}

As a continuation analysis, in this section we focus on how the Pokémon world transcends the game world and affects reality. This enables us to understand in particular the third theme, human relationships and friendship. Ever since the first Pokémon games (see Table 1), players have been encouraged to meet other players in the real world. Motivation for this started as link cable trading and battling, but have since expanded to offline tournaments and other activities. Pokémon GO brought a completely new dimension to the Pokémon world, mixing it with reality using AR features and location-based gameplay [26]. Connecting with other players in the real world brings Pokémon forward as a topic for discussion, and also introduces it as part of playing and imagination [27]. Horton argues that the Pokémon franchise pervasively becomes intertwined with children's spatial and geographical imagination [28]. For example, when engaging in link cable trading players need to barter in real life which pokémon they are going to trade. Pokémon capturing people's imagination is certainly a pervasive phenomenon, and this may be one of the reasons why Pokémon GO became such a commercial success compared to other pervasive games [29].

To some degree, the social hierarchies of the Pokémon world translate into the real world [30]. This is the same phenomenon that is experienced in all sports from football to chess, where those who excel in the sport or game are praised and valued also to a degree outside the game context [30]. The presence of this phenomena motivates people to practise and spend time perfecting their skills and abilities. What makes Pokémon special with this regard is the shared imaginary space that it occupies. This draws parallels to religion where people are united by a shared belief in something that cannot be seen, but perhaps may be felt [31, 32]. While it may be too radical to speak of believing in Pokémon, imagining these creatures in real world environments and discussing them as if they were real invades the human conception of reality [32]. The evolutionary tendency and ability to remember and identify unique pokémon species [33] is a likely contributor as to why Pokémon has such a significant part in children's playing and imagination [28]. Accruing knowledge of the Pokémon world is not game-specific and carries over to new Pokémon games, toys and playing, and even to real world interactions among Pokémon fans.

Pokémon GO in many ways brought the imagination and play of Pokémon fans to reality [34]. Liberati introduces the idea that by situating game objects into the real world, Pokémon GO offers a new way to perceive paramount reality, bringing the Pokémon world as part of our everyday world [26]. Both Liberati and Jensen et al., suggest that players may view Pokémon GO as an additional layer of reality, which can be accessed through mobile devices $[35,26]$. Portraying Pokémon as part of the real world challenges one of the core aspects of the Pokémon franchise: that people in the Pokémon world are altruistic and selfless. The other players in Pokémon GO, being real people, introduced fierce competition at gyms and opened players up for even verbal abuse. Reports came of social injustice and unfair treatment of marginalized individuals [36]. This raises the question of whether the Pokémon world and our world can ever be truly united. However, a recent study by Riar et al., [37] suggests that location-based augmented reality games (e.g. Pokémon GO) may in fact increase altruistic behaviour under specific circumstances. As we strive towards equal and fair treatment of all individuals in our society, games such as Pokémon GO may end up playing a positive role in removing prejudices and supporting marginalized groups.

\section{Comparison with other work and limitations}

Some scholars have claimed that the Pokémon franchise might encourage wildlife exploitation [38]. According to our analysis such claims are unjustified. While pokémon are captured and contained, the Pokémon games, movies and TV-series build a strong narrative highlighting the importance of a loving bond between humans and pokémon. A surprising finding from Butcher et al., [39] was that the alignment of Pokémon GO with the rest of the Pokémon franchise was not significant for the players in their sample, and even more surprisingly, a higher level of nostalgia negatively influenced attitudes towards Pokémon GO [39]. In the light of other studies [5, 13], the findings seem out of place. However, Butcher 
et al., themselves argue that the explanation might be that Pokémon GO does not align with brand quality expectations [40] of long term players [39]. They continue to claim that Pokémon GO sacrificed major story and narrative elements of the Pokémon world in favor of capitalizing on the exploration aspect, which indeed seems to be the case. Based on the thematic analysis of the current study, we argue that Pokémon GO manages to capitalize on some aspects of the Pokémon world such as exploration and freedom of constraints, but fails to support other aspects such as other actors' altruistic behavior.

Besides these two examples, our analysis of the Pokémon world fits well with existing literature (e.g. [8, $15,22,5,2])$. The themes we brought forward slightly differ from those implicitly presented by Elza [22], which were (1) learning; (2) domination; (3) pokémon as loyal allies; and (4) independence/freedom. However, the themes of learning and independence can be connected to exploration, and the theme of pokémon as allies can be connected to the relationship between the trainer and their pokémon. Yet, domination remains an aspect we did not discuss. We acknowledge that the Pokémon world is a vast fictional universe which consists of an abundance of material, some of which is even contradictory with itself. While we consider ourselves experts on the Pokémon franchise, our perspective remains inevitably impacted by our own experiences and standpoint.

\section{Conclusions and Future Work}

In this study we explored four central themes of the Pokémon franchise: (1) relationship with pokémon; (2) exploration and adventure; (3) social connectedness; and (4) green technologies. A cross-cutting theme that united all four was that of reaching harmony with the surrounding environment, technology, people and pokémon. This theme is supported at several levels. First, exploring nature represents going out of the comfort zone while capturing wild pokémon represents containing it [8]. Second, befriending people and pokémon was strongly present in themes 1 and 3. Third, most stories in the Pokémon world include a conflict related to a natural imbalance which the hero or the player needs to resolve by returning the balance. Fourth, the urban planning, technology and architecture in the Pokémon world often depicts balance and harmony between people, pokémon and nature.

While the themes of (1) exploration and adventure; (2) social connectedness; are present in several other popular cross-media franchises such as Harry Potter and Star Wars [41], what remains unique to Pokémon are the pokémon creatures. And while there are franchises that have animal-like creatures such as Digimon and Draconius GO, they lack other aspects of Pokémon such as the theme of seeking harmony and symbiosis with nature. Ultimately it is impossible to pinpoint one or even three aspects about the fictional world of Pokémon that make it stand out as the most profitable cross-media franchise. It is clear that factors such as the gameplay of the main series Pokémon games, the soundtrack of the fist Pokémon movie and gratification from social trading of Pokémon cards have all played a role in the popularity of Pokémon [8, 22]. While such experiences are not constantly repeated, they instill in our consciousness positive memories and experiences we wish to relive, and that manifests as nostalgic feelings towards the franchise.

Moving beyond the Pokémon franchise, our findings encourage research into evolutionary psychology game design that aims to create experiences for people that they find satisfying on a subcortical level. It can be suggested that adventure, exploration, spending time with other lifeforms [7] and seeking symbiosis with nature are things that appeal to humans at a primal level, and appeal to humans universally regardless of social circumstances. These postulates could be the grounds for not only game design, but on the design of stories, movies and books as well.

\section{References}

[1] C. Hallman, "The 25 highest-grossing media franchises of all time," ONLINE, available at: https://www.titlemax.com/discovery-center/money-finance/ the-25-highest-grossing-media-franchises-of-all-timel, visited 6th of June, 2020, 2019.

[2] J. Tobin, Pikachu's global adventure: The rise and fall of Pokémon, Introduction. Duke University Press, 2004.

[3] A. Webster, "Pokémon go spurred an amazing era that continues with sword and shield," The Verge, vol. 28, 2019.

[4] K. Alha, E. Koskinen, J. Paavilainen, and J. Hamari, "Why do people play location-based augmented reality games: A study on pokémon go," Computers in Human Behavior, vol. 93, pp. 114-122, 2019.

[5] J. Hamari, A. Malik, J. Koski, and A. Johri, "Uses and gratifications of pokémon go: why do people play mobile location-based augmented reality games?," International Journal of Human-Computer Interaction, vol. 35, no. 9, pp. 804-819, 2019.

[6] J. M. Vaterlaus, K. Frantz, and T. Robecker, “"reliving my childhood dream of being a pokémon trainer": An exploratory study of college student uses and gratifications related to pokémon go," International Journal of Human-Computer Interaction, vol. 35, no. 7, pp. 596-604, 2019.

[7] A. Balmford, L. Clegg, T. Coulson, and J. Taylor, "Why conservationists should heed pokémon," Science, vol. 295, no. 5564, pp. 2367-2367, 2002. 
[8] A. Allison, Millennial monsters: Japanese toys and the global imagination, vol. 13. Univ of California Press, 2006.

[9] J. Panksepp, Affective neuroscience: The foundations of human and animal emotions. Oxford university press, 2004.

[10] J. Bainbridge, "'it is a pokémon world”: The pokémon franchise and the environment," International Journal of Cultural Studies, vol. 17, no. 4, pp. 399-414, 2014.

[11] D. Buckingham and J. Sefton-Green, "Gotta catch'em all: Structure, agency and pedagogy in children's media culture," Media, Culture \& Society, vol. 25, no. 3, pp. 379-399, 2003.

[12] R. Scully-Blaker, "A practiced practice: Speedrunning through space with de certeau and virilio," Game Studies, vol. 14 , no. 1,2014

[13] D. Harborth and S. Pape, "How nostalgic feelings impact pokémon go players-integrating childhood brand nostalgia into the technology acceptance theory,' Behaviour \& Information Technology, pp. 1-21, 2019.

[14] A. Allison, "The cool brand, affective activism and japanese youth," Theory, Culture \& Society, vol. 26, no. 2-3, pp. 89-111, 2009.

[15] J. Bainbridge, ““'gotta catch "em all!” pokémon, cultural practice and object networks," The IAFOR Journal of Asian Studies, vol. 1, no. 1, pp. 1-15, 2014.

[16] K. Vella, D. Johnson, V. W. S. Cheng, T. Davenport, J. Mitchell, M. Klarkowski, and C. Phillips, "A sense of belonging: Pokemon go and social connectedness," Games and Culture, vol. 14, no. 6, pp. 583-603, 2019.

[17] J. Siegler and J. Loeffler, "The pokémon theme song lyrics," [ONLINE], available at: https://genius.com/Pokemon-pokemon-theme-lyrics, checked 4th of May, 2020, 1999.

[18] P. H. Kahn Jr, "Developmental psychology and the biophilia hypothesis: Children's affiliation with nature," Developmental review, vol. 17, no. 1, pp. 1-61, 1997.

[19] A. Wipper, "The partnership: The horse-rider relationship in eventing," Symbolic Interaction, vol. 23, no. 1 , pp. 47-70, 2000.

[20] C. Appleton and J. Morris, "Scaling skyrim-a case study on the population of solstheim," Journal of Interdisciplinary Science Topics, Volume 7, vol. 7, p. 42, 2018.

[21] S. Laato, T. Pietarinen, S. Rauti, and T. H. Laine, "Analysis of the quality of points of interest in the most popular location-based games," in Proceedings of the 20th International Conference on Computer Systems and Technologies, pp. 153-160, 2019.

[22] C. Elza, "We all live in a pokemon world: animated utopia for kids," The Japanification of Children's Popular Culture: From Godzilla to Miyazaki, edited by Mark West. The Scarecrow Press, Inc, 2009.

[23] S. Kawahara and G. Kumagai, "Expressing evolution in pokémon names: Experimental explorations," Journal of Japanese Linguistics, vol. 35, no. 1, pp. 3-38, 2019.

[24] S. Laato, S. Hyrynsalmi, S. Rauti, and E. Sutinen, "The effects playing pokémon go has on physical activity-a systematic literature review," in Proceedings of the 53rd Hawaii International Conference on System Sciences, 2020.
[25] J. Panksepp, "Cross-species neuroaffective parsing of primal emotional desires and aversions in mammals," Emotion Review, vol. 5, no. 3, pp. 235-240, 2013.

[26] N. Liberati, "Phenomenology, pokémon go, and other augmented reality games," Human studies, vol. 41, no. 2 , pp. 211-232, 2018.

[27] S. Giddings, "Pokémon go as distributed imagination," Mobile Media \& Communication, vol. 5, no. 1, pp. 59-62, 2017.

[28] J. Horton, "'got my shoes, got my pokémon': Everyday geographies of children's popular culture," Geoforum, vol. 43, no. 1, pp. 4-13, 2012.

[29] S. Laato, S. Hyrynsalmi, S. Rauti, A. N. Islam, and T. H. Laine, "Location-based games as exergames-from pokémon to the wizarding world," International Journal of Serious Games, vol. 7, no. 1, pp. 79-95, 2020.

[30] S. Rauti, S. Laato, and T. Pietarinen, "Learning social skills and accruing social capital through pervasive gaming," in Proceedings of DELbA 2020 - Workshop on Designing and Facilitating Educational Location-based Applications, 2020.

[31] H. Gould, "If pokémon go feels like a religion, that's because it kind of is," The Guardian, 2016.

[32] M.-V. Lindsey, "The politics of pokémon. socialized gaming, religious themes and the construction of communal narratives," Online-Heidelberg Journal of Religions on the Internet, vol. 7, 2015.

[33] J. Gomez, M. Barnett, and K. Grill-Spector, "Extensive childhood experience with pokémon suggests eccentricity drives organization of visual cortex," Nature human behaviour, vol. 3, no. 6, pp. 611-624, 2019.

[34] J. Grandinetti and C. Ecenbarger, "Imagine pokémon in the "real" world: a deleuzian approach to pokémon go and augmented reality," Critical Studies in Media Communication, vol. 35, no. 5, pp. 440-454, 2018.

[35] L. J. Jensen, K. D. Valentine, and J. P. Case, "Accessing the pokélayer: Augmented reality and fantastical play in pokémon go," in Educational Media and Technology Yearbook, pp. 87-103, Springer, 2019.

[36] E. K. Layland, G. A. Stone, J. T. Mueller, and C. J. Hodge, "Injustice in mobile leisure: A conceptual exploration of pokémon go," Leisure sciences, vol. 40, no. 4, pp. 288-306, 2018.

[37] M. Riar, B. Morschheuser, J. Hamari, and R. Zarnekow, "How game features give rise to altruism and collective action? implications for cultivating cooperation by gamification," in Proceedings of the 53rd Hawaii International Conference on System Sciences, 2020.

[38] L. J. Dorward, J. C. Mittermeier, C. Sandbrook, and F. Spooner, "Pokémon go: benefits, costs, and lessons for the conservation movement," Conservation Letters, vol. 10, no. 1, pp. 160-165, 2017.

[39] L. Butcher, B. Sung, and K. Raynes-Goldie, "Gotta catch "em all: invigorating pokémon through an innovative brand extension," Journal of Brand Management, vol. 26, no. 3, pp. 227-239, 2019.

[40] G. Yalcinkaya and T. Aktekin, "Brand extension effects and core attributes of experience product franchises: Ab ayesian approach," Journal of Product Innovation Management, vol. 32, no. 5, pp. 731-746, 2015.

[41] C. Taylor, How star wars conquered the universe: the past, present, and future of a multibillion dollar franchise. Hachette UK, 2015. 